\title{
IL-10 promoter genetic polymorphisms and risk of Kawasaki disease in Taiwan
}

\author{
Kai-Sheng Hsieh ${ }^{\mathrm{a}}$, Tsung-Jen Lai ${ }^{\mathrm{b}, \mathrm{c}, \mathrm{d}, 1}$, Yu-Tung Hwang ${ }^{\mathrm{a}, \mathrm{e}}$, Ming-Wei Lin $^{\mathrm{f}}$, Ken-Pen Weng ${ }^{\mathrm{a}, \mathrm{g}}$, \\ Yi-Ten Chiu ${ }^{\text {b,c }}$, Tsyr-Yuh Ho ${ }^{g}$, Chi-Shan Chen ${ }^{\mathrm{a}}$, Yow-Ling Shiue ${ }^{\mathrm{c}}$, Michael Hsiao ${ }^{\mathrm{h}}$, \\ Shih-Feng Tsai ${ }^{\mathrm{i}, \mathrm{j}}$ and Luo-Ping Ger ${ }^{\mathrm{b}, \mathrm{c}, *}$ \\ ${ }^{a}$ Department of Pediatric Cardiology, Kaohsiung Veterans General Hospital, Kaohsiung, Taiwan \\ ${ }^{\mathrm{b}}$ Department of Medical Education and Research, Kaohsiung Veterans General Hospital, Kaohsiung, Taiwan \\ ${ }^{\mathrm{c}}$ Institute of Biomedical Sciences, National Sun Yat-Sen University, Kaohsiung, Taiwan \\ ${ }^{\mathrm{d}}$ Institute of Clinical Medicine, National Yang-Ming University, Taipei, Taiwan

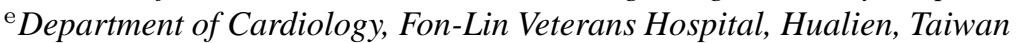 \\ ${ }^{\mathrm{f}}$ Institute of Public Health, National Yang-Ming University, Taipei, Taiwan \\ ${ }^{\mathrm{g}}$ Zuoying Armed Forces General Hospital, Kaohsiung, Taiwan \\ ${ }^{\mathrm{h}}$ Genomic Research Center, Academia Sinica, Taipei, Taiwan \\ ${ }^{i}$ Division of Molecular and Genomic Medicine, National Health Research Institutes, Miaoli, Taiwan \\ ${ }^{\mathrm{j}}$ Department of Life Sciences and Institute of Genome Sciences, National Yang-Ming University, Taipei, Taiwan
}

\begin{abstract}
Kawasaki disease (KD) is the most common cause of pediatric acquired heart disease. KD patients have spontaneously high plasma/serum levels of IL-10 during the acute phase. Therefore, two independent studies were carried out to investigate the association between genetic variants in IL-10 promoter $(-1082,-819$, and -592$)$ and risk of KD. A total of 134 trios were included for the family-based association study. A significantly preferential transmission of the $\mathrm{C}$ allele at loci $-819 \mathrm{~T}>\mathrm{C}$ and $-592 \mathrm{~A}>\mathrm{C}$ for $\mathrm{KD}$ cases was observed $\left(\mathrm{P}_{\text {permutation }}=0.029\right.$ and $\mathrm{P}_{\text {permutation }}=0.034$, respectively). There was a significant increase in the transmission of haplotype CC $(p=0.016)$ at the above two loci (OR, 1.632; 95\%CI, 1.090-2.443; $\mathrm{P}_{\text {permutation }}=$ 0.019). We also carried out a follow-up case-control study that included $146 \mathrm{KD}$ cases and 315 unrelated healthy children. The haplotype CC $(-819,-592)$ showed an increased risk of KD (but statistically non-significant; OR, 1.332; 95\% CI, 0.987-1.797; $p=0.061$ ). In diplotype analysis, a trend was found between number of CC haplotype and risk of KD (but non-significant, $p=$ 0.061). In conclusion, CC genotype and CC/CC diplotype at IL-10-819T $>\mathrm{C}$ and $-592 \mathrm{~A}>\mathrm{C}$ were significantly associated with risk of KD in case-parent trio study, which were replicated partially in our follow-up case-control study.
\end{abstract}

Keywords: Kawasaki disease, risk, IL-10, single-nucleotide polymorphisms, case-parent trio study, and case-control study

\section{Introduction}

Kawasaki disease (KD) is an acute febrile and multisystemic vasculitic syndrome of unknown etiology occurring mostly in children younger than five years of

\footnotetext{
*Corresponding author: Professor Luo-Ping Ger, Department of Medical Education and Research, Kaohsiung Veterans General Hospital, 386 Ta-Chung 1st Road, 813 Kaohsiung, Taiwan. Tel.: +886 7 3468356; Fax: +886 7 3468056; E-mail: lpger@vghks.gov.tw.

${ }^{1}$ Co-first author: Tsung-Jen Lai.
}

age. Coronary artery aneurysms or ectasia occur in up to $30 \%$ of untreated and 5-10\% of treated children [1]. Data has shown that the relative risk of $\mathrm{KD}$ in siblings and twins is 10-fold and 100-fold higher, respectively, as compared to the unaffected Japanese population, suggesting that genetic factors are the underlying cause of KD [2]. The pathogen responsible for the development of $\mathrm{KD}$ is unclear but is generally believed to be an infectious agent. Systemic immune alterations, especially the release of cytokines and chemokines resulting in vascular endothelial inflammation, also play 
a crucial role in the immunopathogenesis of KD [3]. Many studies have demonstrated that the levels of proinflammatory and anti-inflammatory cytokines such as TNF- $\alpha$, IL-1, IL-4, IL-6, IL-8, and IL-10 increase in the acute phase of KD patients [4-7]. For example, KD patients in the acute phase produce nearly 8-to-33-fold and 4-to-5-fold higher plasma or serum levels of IL-10 than healthy controls and acute febrile patients, respectively $[4,8]$. In addition, IL-10 levels elevated during the acute phase of KD and decreased during the subacute and convalescent phases [4] as well as immediately after IVIG administration, which coincides with rapid improvement of inflammatory symptoms [8]. Therefore, the association between genetic variants of IL-10 and risk of $\mathrm{KD}$ is worthy to be evaluated.

Interleukin 10 (IL-10) is an important cytokine with anti-inflammatory and stimulatory activities [9]. It is produced by $\mathrm{T}$ lymphocytes, B lymphocytes, monocytes, and macrophages [10]. IL-10 indirectly inhibits cytokine production by both T cells and NK cells through the inhibition of macrophage and monocyte function [11-14]. Alternatively, IL-10 induces B cell survival, proliferation, and differentiation [15]. In addition, IL-10 enhances immunoglobulin production by naïve and committed B cells and acts as a switch factor for IgG1, IgG3, and IgA production [9]. Levels of IL-10 production are critical in immune regulation and in balancing the inflammatory and humoral responses.

The human IL-10 gene is located on chromosome 1q31-1q32 and composed of five exons and four introns [16]. In the IL-10 gene promoter region, the alleles of $-1082 \mathrm{G},-819 \mathrm{C}$, and $-592 \mathrm{C}$ for three common single nucleotide polymorphisms (SNPs) have been associated with increased production of IL-10 [16-20]. In addition, three previous case-control studies reported inconsistent results for the association of IL-10 genetic polymorphism with risk of KD [18,24,25]. However, the family-based study design, which can avoid the stratification effect on subjects, was not used in the above three studies.

We have previously showed in a prior study that the genotypes and haplotypes with high IL-10 expression were associated with the reduced risk of CALs in the acute stage but not in the chronic stage [23]. In addition, no association between three common SNPs of IL-10 and IVIG resistance was found (not published). Therefore, we hypothesize that the genotypes, haplotypes and diplotypes of IL-10 polymorphisms may have a measurable influence on KD susceptibility. We investigated the possible association of three common promoter SNPs at positions - $1082(\mathrm{~A}>\mathrm{G}),-819(\mathrm{~T}>$ $\mathrm{C})$, and $-592(\mathrm{~A}>\mathrm{C}$ ) with the susceptibility of KD by using a case-parent trio study and a case-control study.

\section{Materials and methods}

Two independent studies (a case-parent trio study and a frequency-matched case-control study) were carried out. KD was diagnosed for patients whose fevers had persisted for at least 5 days and who exhibited at least 4 of the following 5 clinical features, or at least 3 of the clinical features in addition to coronary artery abnormalities detected by echocardiography: 1) bilateral conjunctival congestion, 2) changes to the lips and oral cavity, 3) polymorphous exanthema, 4) changes to the peripheral extremities, and 5) acute nonpurulent cervical lymphadenopathy (Japanese Kawasaki Disease Research Committee, 1984).

\subsection{Study population}

In the case-parent trio study, a total of 138 unrelated KD children who met the standard clinical criteria of KD (Japanese Kawasaki Disease Research Committee, 1984), either met 4/5 clinical criteria (typical KD) or met $3 / 5$ criteria plus coronary artery lesions (atypical $\mathrm{KD}$ ), and their parents were enrolled at the Department of Pediatrics, Kaohsiung Veterans General Hospital (KSVGH), Taiwan. None of the recruited had an individual history of KD. After verifying the family relationships by genotyping another 10 SNPs, four trios that exhibited genotype patterns incompatible with the putative family structure were excluded, yielding 134 trios for analysis.

To validate the results of the initial case-parent trio study, an independent case-control study was performed. A total of $146 \mathrm{KD}$ patients who met the clinical criteria of KD (Japanese Kawasaki Disease Research Committee, 1984), either 4/5 criteria (typical KD) or $3 / 5$ criteria plus coronary artery lesions (atypical KD), were recruited for this study. During the same period, 315 unrelated healthy children who did not have individual history of KD and who were frequency-matched to KD patients on sex and ethnicity were recruited from the Kaohsiung Municipal Da-Yi Junior High School and Kaohsiung Municipal Ding-Jin Junior High School in the KSVGH community.

Information regarding age at onset of $\mathrm{KD}$, sex, selfreported ethnicity, days of fever, response to intravenous immunoglobulin (IVIG), coronary artery status (based on echocardiograms), and laboratory data was extracted from medical records by two senior physicians (Dr. Hwang and Dr. Weng). Questionnaires regarding the demographic data and previous disease histories as well as ethnicity of parents were filled out 
by KD patients' and healthy control children's parents. The protocol was approved by the Institutional Review Board of KSVGH. All parents of subjects gave written informed consent.

\subsection{Polymorphism genotyping}

A total of 875 blood samples were collected for genetic analysis after obtaining informed consent. Genomic DNA for genotyping was extracted and purified from the peripheral blood leukocytes of all subjects using the PUREGENE DNA Purification Kit according to the manufacturer's instructions (Gentra, 2008).

The genotyping of IL-10 single nucleotide polymorphisms (SNPs) at $-1082 \mathrm{~A}>\mathrm{G},-819 \mathrm{~T}>\mathrm{C}$, and $-592 \mathrm{~A}>\mathrm{C}$ was performed by the TaqMan real-time PCR assay using the ABI PRISM 7500 Sequence Detection System (Applied Biosystems, Foster City, CA) in a 96-well format. PCR reactions were carried out in a reaction volume of $10 \mu \mathrm{l}$ containing $20 \mathrm{ng}$ DNA, $5 \mu \mathrm{l} 2 \mathrm{x}$ TaqMan Universal PCR Master Mix (Applied Biosystems), $0.5 \mu \mathrm{l} 20 \mathrm{x}$ Primer/Probe mixture, and $\mathrm{ddH}_{2} \mathrm{O}$ added to a final volume of $10 \mu \mathrm{l}$. The PCR program was as follows: $50^{\circ} \mathrm{C}$ for 2 minutes, $95^{\circ} \mathrm{C}$ for 10 minutes, followed by 40 cycles of 15 seconds at $92^{\circ} \mathrm{C}$ and 1 minute at $60^{\circ} \mathrm{C}$. A no DNA template control (NTC) in each 96-well format was used for quality-control. The allelic-specific fluorescence data from each plate were analyzed using SDS v1.3.1 software (Applied Biosystems, 2005) to determine the genotype of each sample. Quality control procedures are implemented to ensure high genotyping accuracy in our laboratory. Genotyping was repeated for each locus on a 5\% random sampling of the test, and the results were $100 \%$ concordant with the initial analysis. Also, a senior researcher independently reviewed all of the absolute quantification curves of the fluorescence data from the TaqMan assays.

\subsection{Statistical analysis}

For each tested SNP, Hardy-Weinberg equilibrium in KD parents was evaluated by comparing the observed genotype frequencies to the expected genotype frequencies using a goodness of fit chi-square test with one degree of freedom. Pairwise LD $\left(r^{2}\right)$ was computed for all SNPs using the HaploView program (http://www. broad.mit.edu/mpg/haploview/index.php/). The haplotypes and their frequencies were estimated with SAS/Genetics (Version 9.1.3; SAS Institute, Cary, NC, USA) based on the expectation-maximization algo- rithm [26]. For the case-parent trio study, transmission disequilibrium test (TDT) was used to analyze the transmission of alleles from a heterozygous parent to affected offspring. Because there was the possibility of generating false-positive results in TDT, to assess the reliability of the results, permutations $(1,000$ simulations) were performed to generate the empirical $\mathrm{p}$ values. For the case-control study, logistic regression was used to evaluate the association between allelic types, genotypes, and haplotypes of SNPs in IL-10 with KD risk. The odds ratios (OR) and $95 \%$ confidence intervals (CI) were also calculated using logistic regression. To adjust for multiple comparisons, the false discovery rate (FDR) was also computed for each SNP based on the number of SNPs and at-risk allelic type, genotype, and diplotype tested [25]. A $p<0.05$ was considered statistically significant. The SPSS (Version 13.0), SAS/Genetics (Version 9.1.3), gPLINK (Version 2.05), and HaploView (Version 4.1) programs were used for all the statistical analysis.

\section{Results}

\subsection{Association evaluation for case-parent trio study}

A total of 134 trios were recruited for the case-parent trio study. The sex ratio of KD children was 1.81 and their mean age was $2.24 \pm 2.09$ years. The mean age was $2.28 \pm 2.19$ and $2.17 \pm 1.94$ for male and female children with KD, respectively. $85.7 \%$ of the KD children were diagnosed with typical KD and $14.3 \%$ were diagnosed with atypical KD. In addition, $45.5 \%$ of the KD patients had CALs and $9.4 \%$ patients were IVIG resistant.

The association of three IL-10 SNPs, $-1082 \mathrm{~A}>\mathrm{G}$, $-819 \mathrm{~T}>\mathrm{C}$, and $-592 \mathrm{~A}>\mathrm{C}$ with $\mathrm{KD}$ risk was examined by TDT analysis (Table 1 ). There were significant differences in the transmission of allele frequencies for IL-10 -819 T $>\mathrm{C}$ and $-592 \mathrm{~A}>\mathrm{C}(p=$ 0.015 and 0.018 , respectively), even after FDR correction ( $\mathrm{P}_{\text {corrected }}=0.045$ and 0.027 , respectively) and after 1,000 times permutation ( $p=0.029$ and 0.034 , respectively), which corrected for the multiple comparisons made. A significantly preferential transmission of the $\mathrm{C}$ allele at loci $-819 \mathrm{~T}>\mathrm{C}$ and $-592 \mathrm{~A}>\mathrm{C}$ for $\mathrm{KD}$ cases was observed. In addition, a significantly increased risk of KD was found for those with the $\mathrm{C}$ allele at loci $-819 \mathrm{~T}>\mathrm{C}(\mathrm{OR}=1.625,95 \% \mathrm{CI}: 1.10-2.41)$ and $-592 \mathrm{~A}>\mathrm{C}(\mathrm{OR}=1.615,95 \% \mathrm{CI}: 1.08-2.41)$ as compared with each $\mathrm{T}$ and $\mathrm{A}$ allele, respectively. 


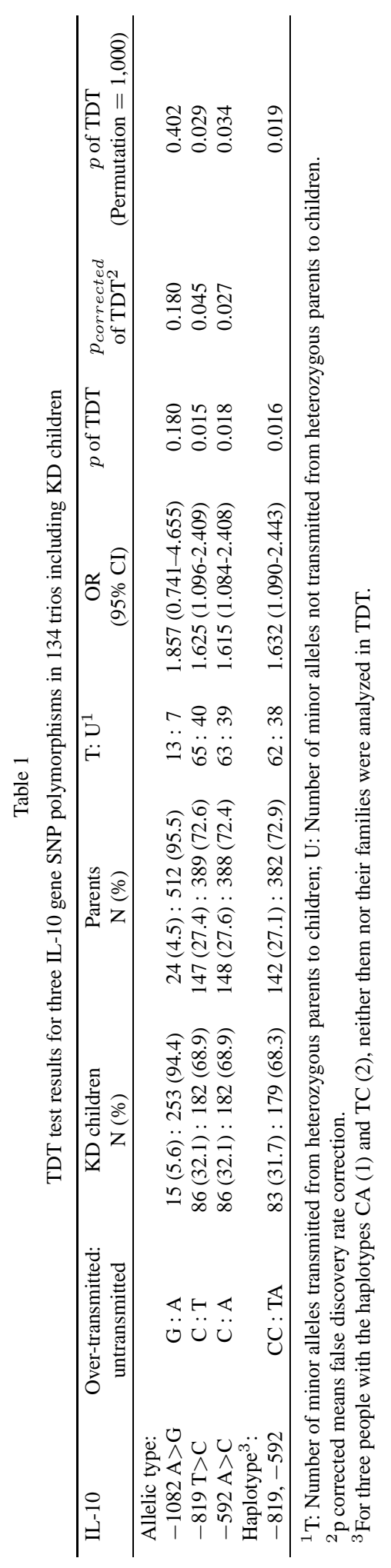


Table 2

Genotypic and allelic distributions of the IL-10 polymorphisms between the KD children and control groups

\begin{tabular}{|c|c|c|c|c|c|c|}
\hline \multirow[t]{2}{*}{ SNP } & \multirow[t]{2}{*}{ Genotype } & KD children & Controls & \multirow[t]{2}{*}{ OR $(95 \% \mathrm{CI})$} & \multirow[t]{2}{*}{ p-value } & \multirow[t]{2}{*}{$p_{\text {corrected }}$} \\
\hline & & $\mathrm{N}(\%)$ & $\mathrm{N}(\%)$ & & & \\
\hline \multirow[t]{7}{*}{$\mathrm{IL}-10-1082 \mathrm{~A}>\mathrm{G}$} & $\mathrm{A} / \mathrm{A}$ & $135(92.5)$ & $283(89.8)$ & 1.000 & & \\
\hline & $\mathrm{A} / \mathrm{G}$ & $11(7.5)$ & $32(10.2)$ & $0.721(0.352-1.473)$ & 0.369 & 0.369 \\
\hline & $\mathrm{G} / \mathrm{G}$ & $0(0)$ & $0(0) \quad]$ & & & \\
\hline & A allele frequency & $281(96.2)$ & $598(94.9)$ & 1.000 & & \\
\hline & $\mathrm{G}$ allele frequency & $11(3.8)$ & $32(5.1)$ & $0.732(0.363-1.472)$ & 0.381 & 0.381 \\
\hline & $\chi^{2}$ value & & 0.902 & & & \\
\hline & $\mathrm{H}-\mathrm{W} p$ value & & 0.342 & & & \\
\hline \multirow{8}{*}{$\mathrm{IL}-10-819 \mathrm{~T}>\mathrm{C}$} & $\mathrm{T} / \mathrm{T}$ & $57(39.0)$ & $154(48.9)$ & 1.000 & & \\
\hline & $\mathrm{T} / \mathrm{C}$ & $73(50.0)$ & $134(42.5)$ & $1.472(0.970-2.233)$ & 0.069 & 0.345 \\
\hline & $\mathrm{C} / \mathrm{C}$ & $16(11.0)$ & $27(8.6)$ & $1.601(0.804-3.189)$ & 0.181 & 0.302 \\
\hline & $\mathrm{T} / \mathrm{C}+\mathrm{C} / \mathrm{C}$ & $89(61.0)$ & $161(51.1)$ & $1.494(1.002-2.226)$ & 0.049 & 0.147 \\
\hline & $\mathrm{T}$ allele frequency & $187(64.0)$ & $442(70.2)$ & 1.000 & & \\
\hline & $\mathrm{C}$ allele frequency & $105(36.0)$ & $188(29.8)$ & $1.320(0.984-1.771)$ & 0.064 & 0.192 \\
\hline & $\chi^{2}$ value & & 0.080 & & & \\
\hline & $\mathrm{H}-\mathrm{W} p$ value & & 0.777 & & & \\
\hline \multirow[t]{8}{*}{$\mathrm{IL}-10-592 \mathrm{~A}>\mathrm{C}$} & $\mathrm{A} / \mathrm{A}$ & $59(40.4)$ & $156(49.5)$ & 1.000 & & \\
\hline & $\mathrm{A} / \mathrm{C}$ & $73(50.0)$ & $134(42.5)$ & $1.440(0.953-2.178)$ & 0.084 & 0.210 \\
\hline & $\mathrm{C} / \mathrm{C}$ & $14(9.6)$ & $25(7.9)$ & $1.481(0.721-3.041)$ & 0.285 & 0.356 \\
\hline & $\mathrm{A} / \mathrm{C}+\mathrm{C} / \mathrm{C}$ & $87(59.6)$ & $159(50.4)$ & $1.447(0.972-2.153)$ & 0.069 & 0.104 \\
\hline & A allele frequency & $191(65.4)$ & $446(70.8)$ & 1.000 & & \\
\hline & $\mathrm{C}$ allele frequency & $101(34.6)$ & $184(29.2)$ & $1.282(0.953-1.723)$ & 0.100 & 0.150 \\
\hline & $\chi^{2}$ value & & 0.260 & & & \\
\hline & $\mathrm{H}-\mathrm{W} p$ value & & 0.610 & & & \\
\hline
\end{tabular}

A weak LD between the SNPs -1082 and -819 $\left(\mathrm{r}^{2}=0.124\right)$ and between the SNPs -1082 and -592 $\left(\mathrm{r}^{2}=0.123\right)$ was found. Strong LD was observed between the SNPs -819 and $-592\left(\mathrm{r}^{2}=0.972\right)$. Therefore, pairwise LD measures $\left(\mathrm{r}^{2}\right)$ across all 3 markers of this gene suggest a distinct haplotype block with only two loci, -819 and -592 , in this region. A haplotypebased TDT was used (Table 1) to test for excessive transmission of certain haplotypes from parents to $\mathrm{KD}$ children. There was a significant increase in the transmission of haplotype CC ( $p=0.016)$, even after 1,000 times permutation $(p=0.019)$. In addition, the haplotype CC was associated with a 1.632-fold (95\% CI: 1.090-2.443) increased risk of KD as compared to the haplotype TA.

\subsection{Association evaluation for case-control study}

The subjects of the case-control study consisted of $146 \mathrm{KD}$ children and 315 sex- and ethnicity-matched healthy children. Among the KD cases, there were 82 males and 64 females with a mean age of $2.37 \pm 2.46$ years. The healthy children consisted of 181 males and 134 females with a mean age of $14.51 \pm 0.50$ years. As expected, the distribution of sex $(\mathrm{M} / \mathrm{F}$ in cases VS. M/F in controls: $82 / 64$ VS. $181 / 134 ; \mathrm{X}^{2}=0.068, p=$ 0.794 ) and ethnicity (Fukienese/Hakka/Mainlander in cases VS. Fukienese/Hakka/Mainlander in controls: $92 / 11 / 19$ VS. $233 / 30 / 52 ; X^{2}=0.096, p=0.951$ ) was comparable between KD cases and healthy children. Among KD cases, $85.6 \%$ had typical KD and $14.4 \%$ had atypical KD. In addition, $32.9 \%$ of KD patients had acute CALs and $12.4 \%$ were IVIG resistant.

The genotypic and allelic frequencies of IL-10 in the case-control study are shown in Table 2. Among controls, the genotype distributions of IL-10 - $1082 \mathrm{~A}$ $>\mathrm{G},-819 \mathrm{~T}>\mathrm{C}$, and $-592 \mathrm{~A}>\mathrm{C}$ were in HardyWeinberg equilibrium (HWE), with $p$ values of 0.342 , 0.777 , and 0.610 , respectively. With regard to genotype of IL-10, no significant association of $-1082 \mathrm{~A}>$ $\mathrm{G},-819 \mathrm{~T}>\mathrm{C}$, and $-592 \mathrm{~A}>\mathrm{C}$ with $\mathrm{KD}$ risk was observed (after FDR $\mathrm{P}_{\text {correction }}=0.150-0.381$ ). An increased risk was found for $\mathrm{C}$ allele $(\mathrm{OR}=1.320,95 \%$ CI: 0.984-1.771), T/C genotype $(\mathrm{OR}=1.472,95 \% \mathrm{CI}$ : 0.970-2.233), or combined T/C $+\mathrm{C} / \mathrm{C}$ genotype (OR $=1.494,95 \% \mathrm{CI}: 1.002-2.226)$ at locus $-819 \mathrm{~T}>\mathrm{C}$ as well as $\mathrm{C}$ allele (OR $=1.282,95 \% \mathrm{CI}: 0.953-1.723)$ and $\mathrm{A} / \mathrm{C}$ genotype $(\mathrm{OR}=1.440,95 \% \mathrm{CI}: 0.953-2.178)$ at locus $-592 \mathrm{~A}>\mathrm{C}$, however, none of them reach any statistical significances after FDR correction. A weak LD between SNPs -1082 and $-819\left(\mathrm{r}^{2}=0.091\right)$, as well as between SNPs -1082 and $-592\left(r^{2}=0.093\right)$, was found. A strong LD was observed between SNPs -819 and $-592\left(\mathrm{r}^{2}=0.977\right)$. Therefore, haplotypes 
Table 3

Results of haplotype and diplotype of IL-10 -819 and -592 polymorphisms between the KD children and control groups

\begin{tabular}{|c|c|c|c|c|c|c|}
\hline \multirow[t]{2}{*}{ IL-10 } & \multirow[t]{2}{*}{ 2-locus SNPs } & KD children & Controls & \multirow[t]{2}{*}{ OR $(95 \% \mathrm{CI})$} & \multirow[t]{2}{*}{ p-value } & \multirow[t]{2}{*}{$p_{\text {corrected }}$} \\
\hline & & $\mathrm{N}(\%)$ & $\mathrm{N}(\%)$ & & & \\
\hline \multirow{2}{*}{ Haplotype $^{1}:-819,-592$} & TA & $186(65.0)$ & $436(71.2)$ & 1.000 & & \\
\hline & $\mathrm{CC}$ & $100(35.0)$ & $176(28.8)$ & $1.332(0.987-1.797)$ & 0.061 & \\
\hline \multirow[t]{4}{*}{ Diplotype $^{2}:-819,-592 /-819,-592$} & $\mathrm{TA} / \mathrm{TA}$ & $57(39.6)$ & $153(49.2)$ & 1.000 & & \\
\hline & $\mathrm{TA} / \mathrm{CC}+\mathrm{TC} / \mathrm{CC}+\mathrm{CA} / \mathrm{CC}$ & $73(50.7)$ & $135(43.4)$ & $1.451(0.957-2.202)$ & 0.080 & 0.159 \\
\hline & $\mathrm{CC} / \mathrm{CC}$ & $14(9.7)$ & $23(7.4)$ & $1.634(0.787-3.393)$ & 0.188 & 0.188 \\
\hline & & & & $p$ for linear trend $=$ & 0.061 & \\
\hline
\end{tabular}

${ }^{1}$ For twelve KD children with the haplotypes CA (10) and TC (2) were not analyzed in the logistic regression.

${ }^{2}$ For six KD children with the diplotypes TC/TC (1), TA/CA (4), and CA/CA (1) were not analyzed in the logistic regression.

$(-819,-592)$ and diplotypes $(-819,-592 /-819$, -592 ) composed of two loci at -819 and -592 were evaluated in relation to the risk of KD. No associations between KD risk and the 2-locus haplotypes and diplotypes were observed. However, the IL-10 haplotype CC was associated with a slightly increased risk of $\mathrm{KD}$ when compared to haplotype TA $(\mathrm{OR}=1.332$, 95\% CI: 0.987-1.797, Table 3). As compared to the diplotype of TA/TA, the combined diplotypes of TA/CC $+\mathrm{TC} / \mathrm{CC}+\mathrm{CA} / \mathrm{CC}$ and diplotype of $\mathrm{CC} / \mathrm{CC}$ had a nonsignificantly increased risk of $\mathrm{KD}(\mathrm{OR}=1.451,95 \%$ CI: 0.957-2.202; OR = 1.634, 95\%CI: 0.787-3.393; respectively). In addition, a linear trend was found between number of $\mathrm{CC}$ haplotype and risk of $\mathrm{KD}$, but statistical significance was not reached $(p=0.061)$.

\section{Discussion}

Our case-parent trio study showed that the $\mathrm{C}$ alleles at IL-10 -819 $\mathrm{T}>\mathrm{C}$ and $-592 \mathrm{~A}>\mathrm{C}$ were transmitted in excess from the parents to the $\mathrm{KD}$ children ( $p=0.029$ and 0.034 , permutation $=1,000$, respectively). In addition, the CC haplotype at the -819 and -592 loci was transmitted in excess from the parents to the KD children $(p=0.019$, permutation $=1,000)$. Our case-control study showed that IL-10 genetic variants were not associated with risk of $\mathrm{KD}$, except haplotype $\mathrm{CC}$ of above two loci with non-significant increased risk of $\mathrm{KD}(p=0.061)$. In addition, a non-significant linear trend ( $p=0.061)$, in the same direction as our trio study, was found between number of CC haplotype and increased risk of $\mathrm{KD}$ in diplotype analysis. Although the findings of our two independent studies were not completely consistent, both of our studies suggested that children carrying the haplotype or diplotype associated with higher levels of IL-10 production had a greater risk of KD.
There have been three case-control studies so far that evaluate IL-10 genetic variation in relation to risk of KD. By a case-control study carried out in Korea by Jin et al. reported similar results to our case-control study. For example, Jin et al. demonstrated that the allele and genotype frequencies of IL-10 - 592 A $>$ C (presented as $-627 \mathrm{~A}>\mathrm{C}$ in their paper) were not associated with KD risk. However, two case-control studies by Yang et al. in southern China and Hsueh et al. in Taiwan showed results contradictory to our case-control study. Yang et al. found that the allelic and three-locus haplotypic $(-1082,-819,-592)$ frequencies of IL-10 were not correlated with susceptibility to KD. Hsueh et al. found that carriers of the -592 A allele or the AA genotype had an increased risk of developing KD. Although all the subjects of these two studies and our study were Han Chinese and the allelic frequencies of these two SNPs $(-819$ and -592$)$ were comparable in the case groups ( $p=0.12-0.52$ ), the allelic frequencies were significantly different in the control groups $(p=$ 0.01-0.03). In our case-control study, ethnic groups were comparable in their distribution between cases and controls, but population stratification can not be ruled out, except in the case-parent trio study.

Our positive findings in the case-parent trio study and weakly positive findings in the case-control study may be explained as follows. First, the case-control study is susceptible to population stratification, which can be rejected in the case-parent trio study because of a common genetic background between KD children and their biological parents. Second, it has been suggested that, for rare diseases, case-parent trio studies are more powerful than case-control studies [26]. The results from both the case-parent trio study and the case-control study only demonstrate small increases in KD risk, which indicate that the IL-10 polymorphisms make only a slight genetic contribution to the development of KD (Tables 1-3). Therefore, the statistical power to detect the association of IL-10 with KD 
in the case-control study design might not be enough. The weak association in our case-control study, such as a non-significant trend in the same direction as our case-parent trio study, may be due to insufficient sample size. Consequently, further replication studies with independent large cohorts are warranted. Third, the environmental factors of case-parent trios tend to be more homogeneous than the environmental factors of casecontrol studies. Fourth, the disease severity or clinical subtypes of KD subjects from the family-based study and the case-control study may be different. The symptoms of the KD patients from the family-based study may be more severe than the symptoms of the KD patients from the case-control study. For example, more acute CALs were identified in the cases $(45.5 \%)$ belonging to our case-parent trio study than those (32.9\%) belonging to our case-control study $\left(\mathrm{X}^{2}=4.70, p=\right.$ $0.03)$.

Three in vitro studies showed consistent results demonstrating that the genetic variation of IL-10 is correlated with IL-10 production. Turner et al. have found that the GCC haplotype is associated with higher IL-10 production compared to other genotypes in PBMC cultures [20]. Crawley et al. also found that PBMCs with either the ATA haplotype or the ATA/ATA diplotype demonstrated decreased transcriptional activity under lipopolysaccharide stimulation compared to other haplotypes (GCC or ACC) or diplotypes [27]. In addition, Edwards-Smith et al. demonstrated that the IL-10 promoter haplotypes (at loci - 1082, -819, and -592) GCC, ACC, and ATA were associated with high, intermediate, and low IL-10 production, respectively [28]. Taken together, these findings support that $\mathrm{C}$ allele, CC haplotype, or CC/CC diplotype of IL-10-819 and -592 are associated with higher levels of IL-10 as compared with other alleles, haplotypes, or diplotype. We previously found that children carrying the higher production genotype $(\mathrm{C} / \mathrm{C})$ or diplotype $(\mathrm{CC} / \mathrm{CC})$ had a lower risk of acute CALs in KD [23]. However, we found that children carrying the higher production genotypes or diplotypes had a higher risk of KD in this study. These results support previous findings demonstrating that KD patients in the acute phase had significantly higher IL-10 plasma or serum levels compared to controls $[4,8]$, and suggest that IL-10 might play a more different role in risk of KD and acute CALs than has been previously supplied. To our knowledge, no other study to date has reported IL-10 to have such apparently opposite effects in susceptibility to KD and CALs. Our findings could supported that KD patients in the acute phase produce higher plasma or serum lev- els of IL-10 than healthy controls $[4,8]$. However, the majority of our KD cases was retrospectively recruited; therefore, we cannot evaluate the relationship between IL-10 plasma levels and genotype during different phases of the disease. Although our study cannot provide functional evidence of the influence of IL-10 polymorphisms on the pathogenesis of $\mathrm{KD}$, the higher IL-10 production genotype $\mathrm{C} / \mathrm{C}$ and diplotype $\mathrm{CC} / \mathrm{CC}$ (C allele over-transmitted from both father and mother) at loci $-819 \mathrm{~T}>\mathrm{C}$ and $-592 \mathrm{~A}>\mathrm{C}$ were associated with the development of KD in our study. Thus, both of them contributed slightly (mild increment of ORs in Tables 1-3) to KD risk in this study. We suggest that other important candidate genes may predispose children to KD, and they will need to be identified in the near future.

IL-10 has multiple effects on antigen-presenting cell (APC), T cell, and B cell. In the acute phase of KD patients, the massive immune activation mediated by pathogen lead to marked cytokine production, such as IL-1, IL-2, IL-6, IL-8, interferon- $\gamma$, and TNF- $\alpha$ from monocytes, macrophages, and Th1 cells [31,32]. In this condition, the marked increased IL-10 may suppress the pro-inflammatory cytokines to balance the serious immune response [4,8,9,33]. In addition, IL-10 strongly inhibits the proliferation of Th0 cells and activation of Th1 cells by down-regulating the antigen-present of APC [9]. Th1 cells are essential for effective cellmediated immunity. Therefore, the bacterial clearance will be down-regulated by inhibiting the cell-mediated immunity. Base on above evidence or proposed events, higher IL-10 may increase the risk of KD by inhibiting bacterial clearance. But it may decrease the risk of acute CAL by inhibiting TNF- $\alpha$ and IL- 1 which are necessary for both inflammation and vascular damage during the development of coronary artery aneurysms in an animal model of KD [23]. On the other hand, IL10 also stimulates $\mathrm{B}$ cell proliferation, differentiation, and immunoglobulin production. Infiltration of arterial walls and the upper respiratory tract by $\operatorname{IgA}$ plasma cells occurred in acute KD patients [29,30], which may be due to IL-10 stimulation of $\mathrm{B}$ cells and the transformation of $\mathrm{B}$ cells into IgA plasma-cells. In addition, IL-10 enhances immunoglobulin production by naïve and committed $B$ cells and acts as a switch factor for $\operatorname{IgG} 1, \operatorname{IgG} 3$ and $\operatorname{Ig}$ A production [11], which may increase the risk of KD by stimulating $B$ cell and inducing IgA-plasma cell infiltration. Although the role of IL-10 in the etiology of KD still not clear, all the related studies suggested that the increased levels of serum IL-10 may have an effect on immune responses 
by inhibiting pro-inflammatory cytokine synthesis and by stimulating $\mathrm{B}$ cells proliferation, differentiation, and IgA production during acute phase of $\mathrm{KD}[4,8,33]$.

In conclusion, the $\mathrm{CC}$ genotype and $\mathrm{CC} / \mathrm{CC}$ diplotype at IL-10-819T $>\mathrm{C}$ and $-592 \mathrm{~A}>\mathrm{C}$ were significantly associated with risk of KD in the case-parent trio study. However, this finding was only partially replicated in the follow-up case-control study because of limited statistical power and the smallness of the effect of the IL-10 gene on KD risk. Therefore, we suggest that IL-10 genetic polymorphism might be a protective factor against sequelae of CALs but not a risk factor for $\mathrm{KD}$, except an independent cohort study confirm the significance of our findings.

\section{Acknowledgments}

We thank Mrs. Hsiu-Hsing Tsai and Mrs. Hui-Wen Huang for helpful control samples collection from the Kaohsiung Municipal Da-Yi Junior High School and Kaohsiung Municipal Ding-Jin Junior High School, and Miss Ching-Ju Hsu in the Department of Pediatric Cardiology, Kaohsiung Veterans General Hospital for helpful KD and parents samples collection. Segments of this research were supported by the Kaohsiung Veterans General Hospital (VGHKS96-111 and VGHKS98084), VTY Joint Research Program (VGHUST95-G303-2, VGHUST97-G3-3 and VGHUST98-G3-2), and Zuoying Armed Forces Hospital (9810 and 9811). We also thank Christina Jen-Chia Hsieh for assistance in English editing.

\section{References}

[1] D. Burgner and A. Harnden, Kawasaki disease: what is the epidemiology telling us about the etiology? Int J Infect Dis 9 (2005), 185-194.

[2] Y. Fujita, Y. Nakamura, K. Sakata, N. Hara, M. Kobayashi and M. Nagai et al., Kawasaki disease in families, Pediatrics 84 (1989), 666-669.

[3] C.L. Wang, Y.T. Wu, C.A. Liu, H.C. Kuo and K.D. Yang, Kawasaki disease: infection, immunity and genetics, Pediatr Infect Dis J 24 (2005), 998-1004.

[4] D.S. Kim, H.K. Lee, G.W. Noh, S.I. Lee and K.Y. Lee, Increased serum interleukin-10 level in Kawasaki disease, Yonsei Medical Journal 37 (1996), 125-130.

[5] C.Y. Lin, C.C. Lin, B. Hwang and B. Chiang, Serial changes of serum interleukin-6, interleukin-8, and tumor necrosis factor alpha among patients with Kawasaki disease, The Journal of Pediatrics 121 (1992), 924-926.

[6] C.P. Maury, E. Salo and P. Pelkonen, Circulating interleukin1 beta in patients with Kawasaki disease, The New England Journal of Medicine 319 (1988), 1670-1671.
[7] H. Tsukahara, M. Hiraoka, M. Saito, K. Nishida, R. Kobata, S. Tsuchida, M. Toyooka, H. Kimura, F. Gejyo and M. Mayumi, Methylenetetrahydrofolate reductase polymorphism in Kawasaki disease, Pediatr Int 42 (2000), 236-240.

[8] G.W. Noh, W.G. Lee, W. Lee and K. Lee, Effects of intravenous immunoglobulin on plasma interleukin-10 levels in Kawasaki disease, Immunology letters 62 (1998), 19-24.

[9] K.W. Moore, R. de Waal Malefyt, R.L. Coffman and A. O'Garra, Interleukin-10 and the interleukin-10 receptor, Annual Review of Immunology 19 (2001), 683-765.

[10] T.R. Mosmann, Properties and functions of interleukin-10, Advances in Immunology 56 (1994), 1-26.

[11] R. de Waal Malefyt, J. Abrams, B. Bennett, C.G. Figdor and J.E. de Vries, Interleukin 10 (IL-10) inhibits cytokine synthesis by human monocytes: an autoregulatory role of IL-10 produced by monocytes, The Journal of Experimental Medicine 174 (1991), 1209-1220.

[12] L. Ding and E.M. Shevach, IL-10 inhibits mitogen-induced $\mathrm{T}$ cell proliferation by selectively inhibiting macrophage costimulatory function, J Immunol 148 (1992), 3133-3139.

[13] D.F. Fiorentino, A. Zlotnik, T.R. Mosmann, M. Howard and A. O'Garra, IL-10 inhibits cytokine production by activated macrophages, J Immunol 147 (1991), 3815-3822.

[14] D.H. Hsu, K.W. Moore and H. Spits, Differential effects of IL-4 and IL-10 on IL-2-induced IFN-gamma synthesis and lymphokine-activated killer activity, International Immunology 4 (1992), 563-569.

[15] K. Itoh and S. Hirohata, The role of IL-10 in human B cell activation, proliferation, and differentiation, J Immunol 154 (1995), 4341-4350.

[16] J. Eskdale, D. Kube, H. Tesch and G. Gallagher, Mapping of the human IL10 gene and further characterization of the 5' flanking sequence, Immunogenetics 46 (1997), 120-128.

[17] J. Eskdale, G. Gallagher, C.L. Verweij, V. Keijsers, R.G. Westendorp and T.W. Huizinga, Interleukin 10 secretion in relation to human IL-10 locus haplotypes, Proceedings of the National Academy of Sciences of the United States of America 95 (1998), 9465-9470.

[18] H.S. Jin, H.B. Kim, B.S. Kim, J.K. Lee, E.J. Seo, H.W. Yoo, I.S. Park, Y.M. Hong and S.J. Hong, The IL-10 (-627 A/C) promoter polymorphism may be associated with coronary aneurysms and low serum albumin in Korean children with Kawasaki disease, Pediatric Research 61 (2007), 584-587.

[19] K. Koss, J. Satsangi, G.C. Fanning, K.I. Welsh and D.P. Jewell, Cytokine (TNF alpha, LT alpha and IL-10) polymorphisms in inflammatory bowel diseases and normal controls: differential effects on production and allele frequencies, Genes and Immunity 1 (2000), 185-190.

[20] D.M. Turner, D.M. Williams, D. Sankaran, M. Lazarus, P.J. Sinnott and I.V. Hutchinson, An investigation of polymorphism in the interleukin-10 gene promoter, Eur J Immunogenet 24 (1997), 1-8.

[21] J. Yang, C.R. Li, Y.B. Li, R.X. Li, L.B. Sun, H.J. Huang and G.B. Wang, The correlation between Kawasaki disease and polymorphisms of Tumor necrosis factor alpha and interleukin-10 gene promoter, Zhonghua er ke za zhi 41 (2003), 598-602.

[22] K.C. Hsueh, Y.J. Lin, J.S. Chang, L. Wan, Y.H. Tsai, C.H. Tsai, C.P. Chen and F.J. Tsai, Association of interleukin-10 A-592C polymorphism in Taiwanese children with Kawasaki disease, Journal of Korean Medical Science 24 (2009), 438-442.

[23] K.P. Weng, K.S. Hsieh, Y.T. Hwang, S.H. Huang, T.J. Lai, Y.S. Yuh, Y.Y. Hou, C.C. Lin, S.C. Huang and C.K. Chang, IL-10 
polymorphisms are associated with coronary artery lesions in acute stage of Kawasaki disease, Circ J 74 983-989.

[24] H. Zhao, J. Li and W.P. Robinson, Multipoint genetic mapping with uniparental disomy data, American Journal of Human Genetics 67 (2000), 851-861.

[25] Y. Benjamini, D. Drai, G. Elmer, N. Kafkafi and I. Golani, Controlling the false discovery rate in behavior genetics research, Behav Brain Res 125 (2001), 279-284

[26] N.M. Laird and C. Lange, Family-based designs in the age of large-scale gene-association studies, Nature Reviews 7 (2006), 385-394.

[27] E. Crawley, R. Kay, J. Sillibourne, P. Patel, I. Hutchinson and P. Woo, Polymorphic haplotypes of the interleukin-10 5' flanking region determine variable interleukin-10 transcription and are associated with particular phenotypes of juvenile rheumatoid arthritis, Arthritis and Rheumatism 42 (1999), 1101-1108.

[28] C.J. Edwards-Smith, J.R. Jonsson, D.M. Purdie, A. Bansal, C. Shorthouse and E.E. Powell, Interleukin-10 promoter polymorphism predicts initial response of chronic hepatitis $\mathrm{C}$ to interferon alfa, Hepatology 30 (1999), 526-530.

[29] A.H. Rowley, S.T. Shulman, C.A. Mask, L.S. Finn, M. Terai,
S.C. Baker, C.A. Galliani, K. Takahashi, S. Naoe, M.B. Kalelkar and S.E. Crawford, IgA plasma cell infiltration of proximal respiratory tract, pancreas, kidney, and coronary artery in acute Kawasaki disease, The Journal of Infectious Diseases 182 (2000), 1183-1191.

[30] A.H. Rowley, S.T. Shulman, B.T. Spike, C.A. Mask and S.C. Baker, Oligoclonal $\mathrm{IgA}$ response in the vascular wall in acute Kawasaki disease, J Immunol 166 (2001), 1334-1343.

[31] S. Furukawa, T. Matsubara, K. Jujoh, K. Yone, T. Sugawara, K. Sasai, H. Kato and K. Yabuta, Peripheral blood monocyte/macrophages and serum tumor necrosis factor in Kawasaki disease, Clin Immunol Immunopathol 48 (1988), 247-251.

[32] Y. Ueno, N. Takano, H. Kanegane, T. Yokoi, A. Yachie, T. Miyawaki and N. Taniguchi, The acute-phase nature of interleukin 6: studies in Kawasaki disease and other febrile illnesses, Clin Exp Immunol 76 (1989), 337-342.

[33] Y. Okada, M. Shinohara, T. Kobayashi, Y. Inoue, T. Tomomasa, T. Kobayashi and A. Morikawa, Effect of corticosteroids in addition to intravenous gamma globulin therapy on serum cytokine levels in the acute phase of Kawasaki disease in children, J Pediatr 143 (2003), 363-367. 


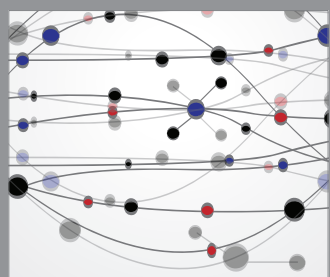

The Scientific World Journal
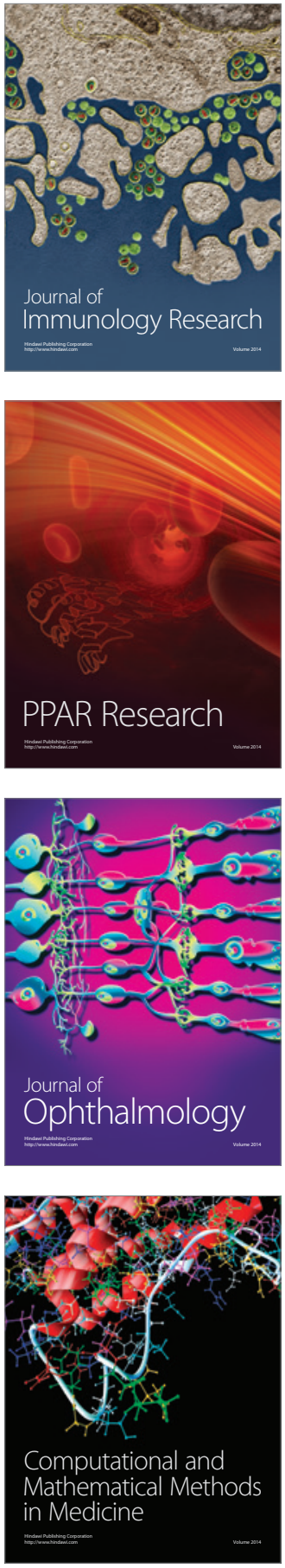

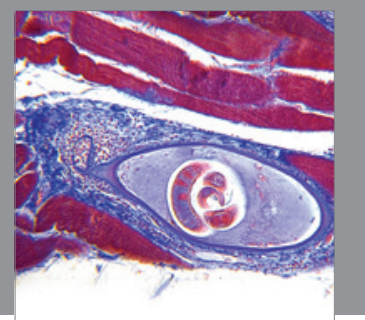

Gastroenterology

Research and Practice
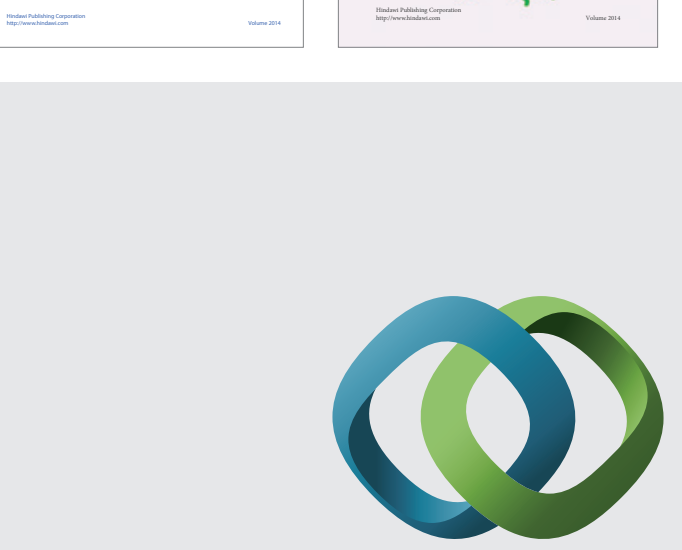

\section{Hindawi}

Submit your manuscripts at

http://www.hindawi.com
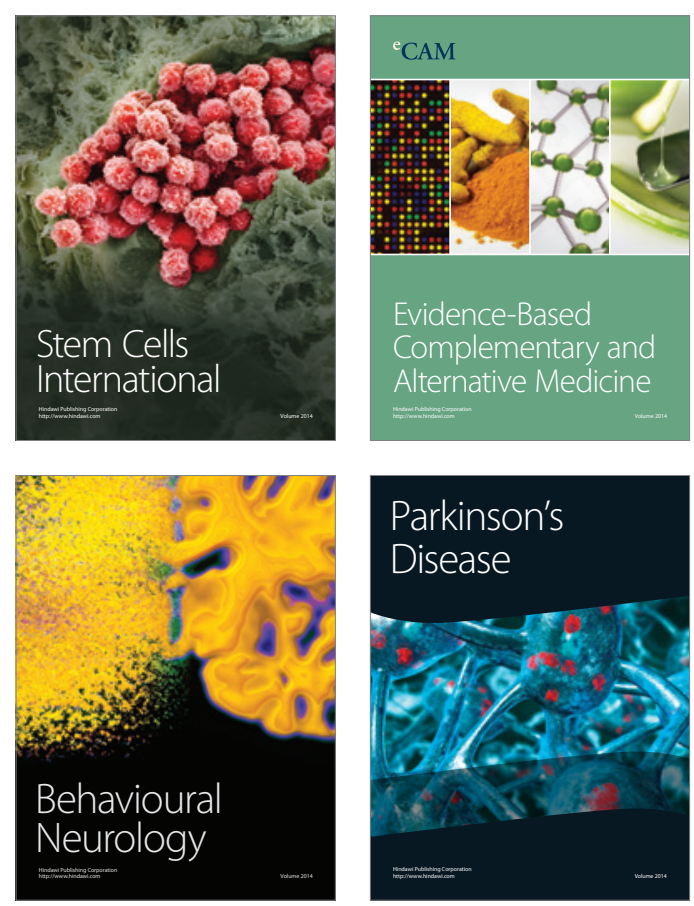

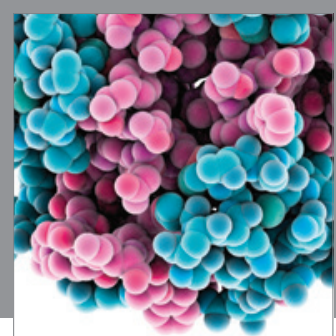

Journal of
Diabetes Research

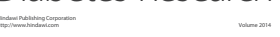

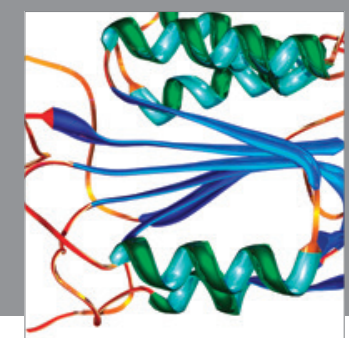

Disease Markers
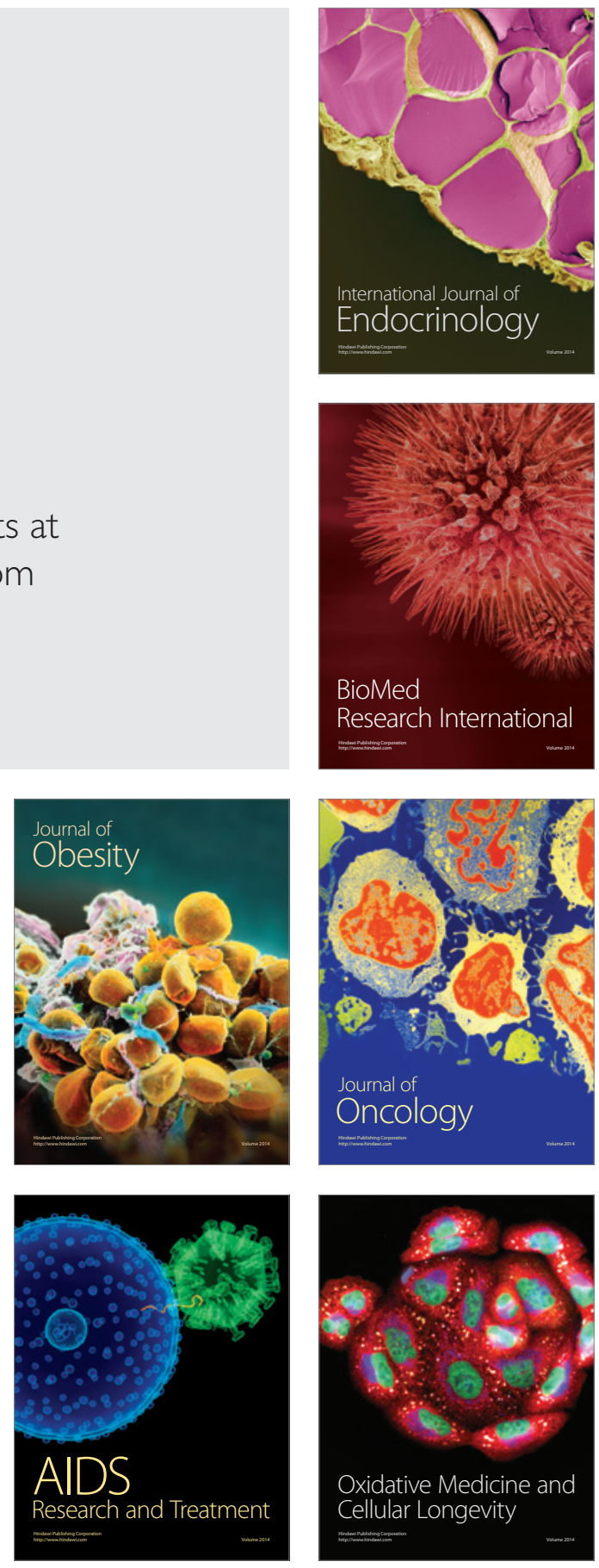